Discrete Comput Geom 30:241-262 (2003)

DOI: $10.1007 / \mathrm{s} 00454-003-0007-6$

\title{
Some Densest Two-Size Disc Packings in the Plane*
}

\author{
Aladár Heppes \\ Vércse u. 24/A, 1124 Budapest, Hungary \\ hep9202@helka.iif.hu
}

Dedicated to László Fejes Tóth on his $85^{\text {th }}$ birthday

\begin{abstract}
The main purpose of this paper is to prove some long-standing conjectures concerning the packing density of some compact arrangements of discs of two different radii in the Euclidean plane. To reach this goal a new method, called cell balancing, is presented.
\end{abstract}

\section{Introduction}

In this paper two-size disc packings in the Euclidean plane are studied, disc packings consisting of copies of two given discs. The main purpose of this paper is to determine the maximal density of such a packing in six particular cases, when the conjectured best arrangement is a "compact packing" [FeL3]. (The reader is reminded that in a compact packing each disc is tangent to a ring of finitely many other discs, each of which is tangent to two further ones of the ring in cyclic order such that the convex hull of the centers of the neighbors contains the center of the first disc.)

It has been known for about a century [Th1], [Th2] that the density of a packing of congruent discs cannot exceed $\delta_{0}=\pi / \sqrt{12}=0.9069 \ldots$, the ratio of the area of a circle and that of a circumscribed regular hexagon. The problem of determining the largest possible density of packings consisting of incongruent discs was first raised in Lagerungen. . . [FeL1], the well-known monograph of L. Fejes Tóth. From the numerous results of this topic, given in the references, we mention only those having direct consequences on two-size disc packings.

\footnotetext{
* This research has been supported in part by the Hungarian research foundation OTKA, Grant Numbers T030012 and T037752.
} 
The upper bounds given by L. Fejes Tóth and Molnár [FM] for the least upper bound $\delta(1, r)$ of the density of a packing of unit discs and discs of radius $r<1$ have been sharpened by Florian [Fl1], who proved that the density cannot exceed the packing density within a triangle determined by the centers of mutually touching circles of radius $1, r$ and $r$. Unfortunately, such packings do not tile the plane for any value of $r$, thus this general bound is never sharp.

We know more about the two ends of the $r$ interval. L. Fejes Tóth noticed on the one hand that $\delta(1, r)<1-\left(1-\delta_{0}\right)^{2}=0,9913 \ldots$ and $\lim _{r \rightarrow 0} \delta(1, r)=1-\left(1-\delta_{0}\right)^{2}$, and on the other hand that the arrangement of equal discs is so efficient that its density $\delta_{0}$ cannot be improved by the use of discs just slightly smaller than the unit disc.

The latter result started another direction of investigation. Florian [Fl2], extended L. Fejes Tóth's interval (in which $\left.\delta(1, r)=\delta_{0}\right)$ to $(0.906 \ldots, 1]$, then, based on an idea of Böröczky [Bö], independent proofs have been published by Blind [Bl] and G. Fejes Tóth [FeG] implying that whenever $r>0.742 \ldots$, the density of a packing of unit discs and discs of radius $r$ is $\leq \pi / \sqrt{12}$. In other words, in this relatively large interval you cannot do better than use equal discs.

In the rest of the $r$ interval many nice and promising packings (see, e.g. [FeL1], $[\mathrm{HM}]$ or [FeL2]) have been constructed (mainly by Molnár [Mo]), some of them with a density very close to Florian's upper bound, but, until recently, no effort yielded a densest packing of incongruent discs. In 1999 the author succeeded in proving that the system of incircles of the Archimedean mosaic $(4,8,8)$ is the densest packing of unit discs and discs of radius $\sqrt{2}-1[\mathrm{He}]$. The method used there needed essential extensions to handle other cases of compact packings and now we are able to raise the number of the solved cases to six. The extremal packings or target packings, the radius of the small disc in them and the packing density will be denoted by $\mathcal{P}_{k}, r(k)$ and $\delta^{*}(k)$, respectively. (The extremal packings are illustrated in Figs. 1-6. The lines indicate the structure of the triangulation used in the proof).

Our main goal is to prove the following:

Theorem. The density of a packing consisting of discs of radius 1 and discs of radius $r(k)$ is at most $\delta^{*}(k)$, the density of the packing $\mathcal{P}_{k}, k=1, \ldots, 6$, i.e. $\delta(1, r(k))=\delta^{*}(k)$.

The description of the six target packings mentioned in the Theorem is given below:

In $\mathcal{P}_{1}$ a pair of small discs in tangent position is surrounded by a ring of six unit discs (see Fig. 1).

In $\mathcal{P}_{2}$ tangent unit discs form parallel straight strings separated by small discs in such a way that each unit disc has two large and six small neighbors (see Fig. 2).

In $\mathcal{P}_{3}$ the discs are the incircles of the Archimedean tiling $(4,8,8)$ of suitable size (see Fig. 3).

In $\mathcal{P}_{4}$ the small discs are centered at the vertices of an Archimedean tiling $(3,12,12)$ of edge length $2 r$ and 12 small discs form a ring around each unit disc (see Fig. 4).

In $\mathcal{P}_{5}$ a pair of small discs in tangent position is surrounded by a ring of four unit discs (see Fig. 5).

In $\mathcal{P}_{6}$ the discs are the incircles of the Archimedean tiling $(3,12,12)$ of suitable size (see Fig. 6). 


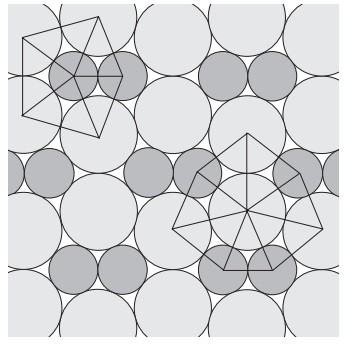

Fig. 1. $\mathcal{P}_{1}$.

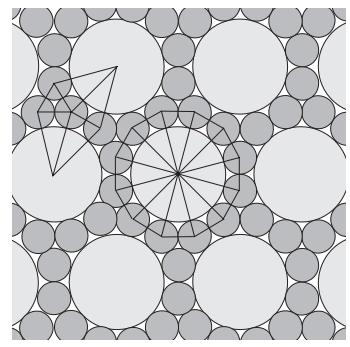

Fig. 4. $\mathcal{P}_{4}$.

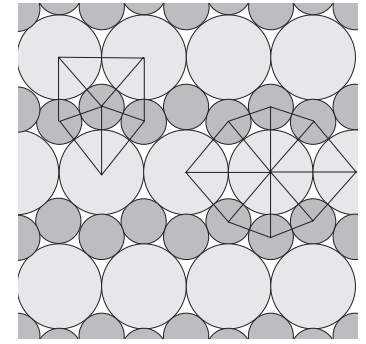

Fig. 2. $\mathcal{P}_{2}$.

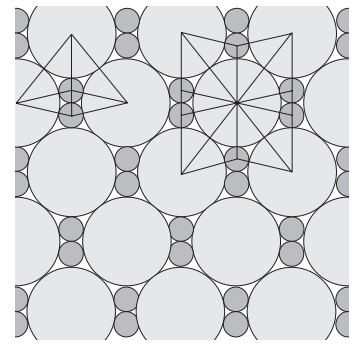

Fig. 5. $\mathcal{P}_{5}$.

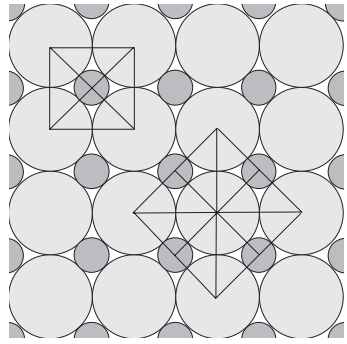

Fig. 3. $\mathcal{P}_{3}$.

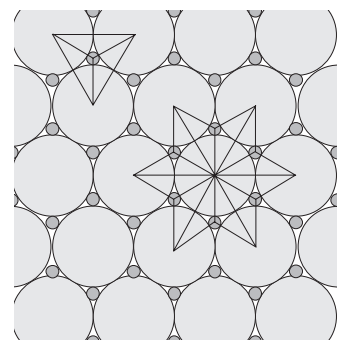

Fig. 6. $\mathcal{P}_{6}$.

Remarks. (1) These densest arrangements are clearly not unique, as the density of a packing is not affected by changes confined to a bounded part of the plane.

(2) The method developed to prove the Theorem is based on the decomposition of the plane into cells in such a way that the density does not exceed the density of the target packing in any cell. To build the required cells each triangle of the marked FMtriangulation (see below) of the saturated packing considered is cut into three pieces first, then sets of pieces sharing a common vertex are united to form "balanced" cells. It is the decomposition rule applied to the triangles which makes the cells balanced and the idea work.

(3) The method is quite flexible and it is expected to be useful in other applications as well. It is suitable to prove, e.g. that the $\delta(1, r)$ function has strict local maxima at $r_{i}, i=1, \ldots, 6$, and Florian's general upper bound can be improved in many cases, especially $\delta(1,0.6)=\delta(1,0.67)=\delta_{0}$. An important consequence of this is that $d(1, r)$ is not monotonous. However, to keep the long proof as simple as possible these other applications are not included in this paper.

The known results—including the ones mentioned in the above paragraph-are illustrated in Fig. 7.

(4) The extremality of the arrangements in the Theorem can be proved even among weighted packings where the specific weights of the discs of different radii differ slightly.

\section{Notations}

We remind the reader that a packing is called saturated with discs of radius $r$ if no further disc of radius $r$ can be added to it without violating its packing property. Seeking maximal 


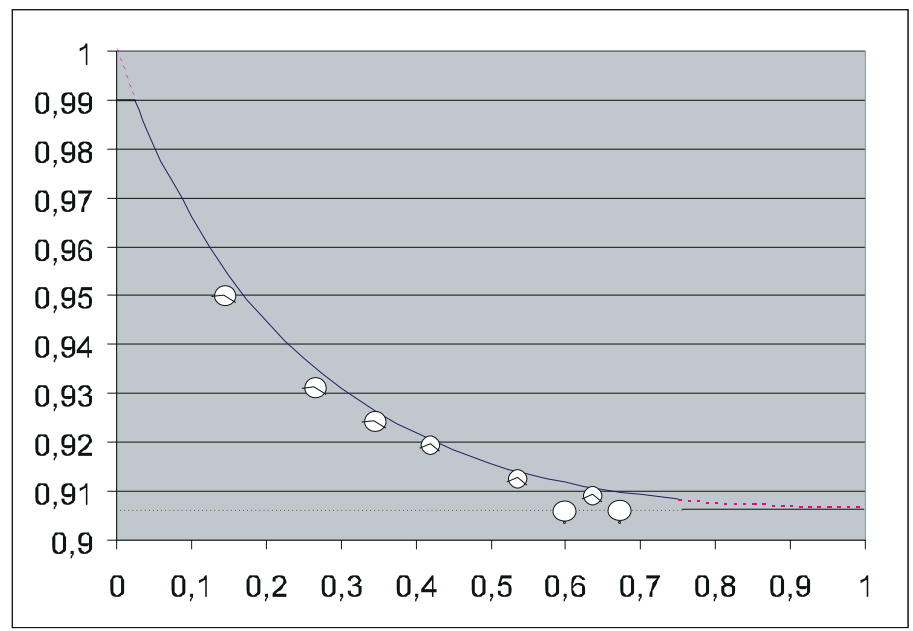

Fig. 7

density it can be assumed without loss of the generality that the packings studied are saturated with discs of radius $r$, or saturated, in short.

By FM-triangulation of a saturated packing we mean a certain subdivision of the plane into triangles such that the set of vertices of the triangles is the set of the centers of the discs (see [FM] or pp. 183-198 in [FeL2]). To generate an FM-triangulation consider all circles which are disjoint from the interior of all discs of the packing, but tangent to at least three of them. These are the support circles of the packing. The centers of the (at least three) discs which are in contact with a particular support circle define a polygon. These polygons generate a decomposition of the plane. If a polygon has more than three sides, it will be further partitioned into triangles by non-crossing diagonals chosen at will. The FM-triangulation has the property that the disc sectors defined by the sides of a triangle lie in the triangle completely, i.e. each disc is covered by the triangles meeting at its center. By marking each vertex of the triangles with the radius of the disc centered at it we get a marked FM-triangulation.

Saturation of the packing ensures that the radius of the support circle touching the three discs around the vertices of the triangle is smaller than $r$. In the discussion we usually assume that the triangles are feasible, i.e. they could occur in the marked FM-triangulation of a saturated packing of admissible discs, and that adjacent marked triangles join properly, i.e. shared vertices bear the same marking.

A triangle is called tight if its vertices are centers of three mutually tangent discs. (See Fig. 8(a).) A compact packing decomposes in a natural way (by connecting the centers of touching discs) into tight triangles (see Figs. 1-6). In a two-size packing of discs four types of tight triangles can occur. We say that a triangle is of type $i, i=1, \ldots, 4$, if the number of unit discs and small discs among the discs at the vertices of the triangle is $4-i$ and $i-1$, respectively:

$\mathcal{P}_{1}$ (Fig. 1) splits into type 2 and type 3 tight triangles.

$\mathcal{P}_{2}$ (Fig. 2) splits into type 2 and type 3 tight triangles. 




(a)

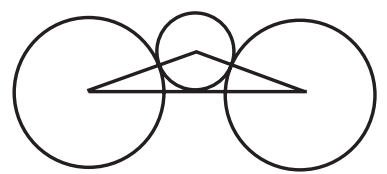

(b)

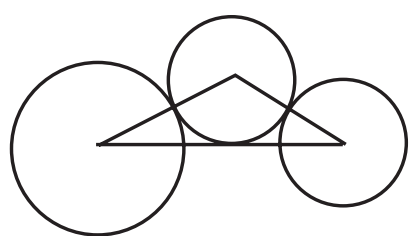

(c)

Fig. 8

$\mathcal{P}_{3}$ (Fig. 3) splits into type 2 tight triangles.

$\mathcal{P}_{4}$ (Fig. 4) splits into type 3 and type 4 tight triangles.

$\mathcal{P}_{5}$ (Fig. 5) splits into type 2 and type 3 tight triangles.

$\mathcal{P}_{6}$ (Fig. 6) splits into type 2 tight triangles.

The density in a tight triangle of type $i$ is denoted by $\delta_{i}(k), k=1, \ldots, 6$. The different density values related to the six target packings are listed in Table 1 .

A triangle is called stretched if its vertices are the centers of a small disc and two other discs of admissible radii such that the small disc is touching the two other discs and the opposite side of the triangle defined by the centers. (See Fig. 8(b),(c).) Clearly, a stretched triangle is not feasible since its support circle would be of radius $r$, however, it can be approached arbitrarily by feasible triangles.

A function $f$ is said to be quasi-concave on an interval $I$ if

$$
f(\lambda x+(1-\lambda) y) \geq \min \{f(x), f(y)\}
$$

for any $x, y \in I$ and all $\lambda \in(0,1)$.

For the four types (or classes) of feasible triangles defined by the admissible radii 1 and $r$ we also use the notation $T[1,1,1], T[1,1, r], T[1, r, r]$ and $T[r, r, r]$, where the radii are listed in the brackets. Subsets of each class will be characterized by the touching number, the number of pairs of discs in contact. The touching number is given as subscript of $T$, and the arguments are listed so that the order of them indicates the touching pairs:

$T_{1}\left[\varrho_{1}, \varrho_{2}, \varrho_{3}\right]$ is the subclass of triangles with the property that the disc of radius $\varrho_{1}$ and that of radius $\varrho_{2}$ are in contact $\left(T_{1}\left[\varrho_{1}, \varrho_{2}, \varrho_{3}\right] \equiv T_{1}\left[\varrho_{2}, \varrho_{1}, \varrho_{3}\right]\right)$. In $T_{2}\left[\varrho_{1}, \varrho_{2}, \varrho_{3}\right]$

Table 1. Density values for the different target packings.

\begin{tabular}{ccccccc}
\hline & $\mathcal{P}_{1}$ & $\mathcal{P}_{2}$ & $\mathcal{P}_{3}$ & $\mathcal{P}_{4}$ & $\mathcal{P}_{5}$ & $\mathcal{P}_{6}$ \\
\hline$r=$ & $0.637555 \ldots$ & $0.533296 \ldots$ & $0.414213 \ldots$ & $0.349198 \ldots$ & $0.280776 \ldots$ & $0.154700 \ldots$ \\
$\delta_{2}=$ & $0.910661 \ldots$ & $0.914020 \ldots$ & $0.920151 \ldots$ & $0.924969 \ldots$ & $0.931615 \ldots$ & $0.950307 \ldots$ \\
$\delta_{3}=$ & $0.910770 \ldots$ & $0.914300 \ldots$ & $0.920835 \ldots$ & $0.926021 \ldots$ & $0.933207 \ldots$ & $0.953299 \ldots$ \\
$\delta^{*}=$ & $0.910683 \ldots$ & $0.914179 \ldots$ & $0.920151 \ldots$ & $0.924648 \ldots$ & $0.931901 \ldots$ & $0.950307 \ldots$ \\
\hline
\end{tabular}



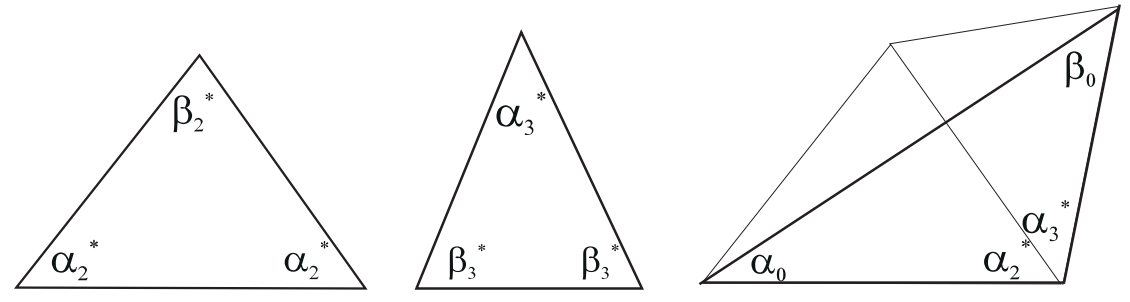

Fig. 9

there are two pairs of touching discs, the disc of radius $\varrho_{2}$ is in contact with the other two discs $\left(T_{2}\left[\varrho_{1}, \varrho_{2}, \varrho_{3}\right] \equiv T_{2}\left[\varrho_{3}, \varrho_{2}, \varrho_{1}\right]\right)$. With respect to the symmetry, the number of different $T_{1}, T_{2}$ and $T_{3}$ subclasses is 6,6 and 4 , respectively. Clearly, $T_{3}\left[\varrho_{1}, \varrho_{2}, \varrho_{3}\right] \subset$ $T_{2}\left[\varrho_{1}, \varrho_{2}, \varrho_{3}\right] \subset T_{1}\left[\varrho_{1}, \varrho_{2}, \varrho_{3}\right]$.

In general, $A$ or $A_{i}$ and $\alpha$ or $\alpha_{i}$ are used to denote the vertex of a triangle if it is the center of a unit disc and the angle of the triangle at this vertex, respectively, while $B$ or $B_{i}$ and $\beta$ or $\beta_{i}$ are used in connection with a disc of radius $r$. We denote the segment connecting points $X$ and $Y$ as well as its length by $X Y$.

It will be practical to predefine a few constants (see Fig. 9):

$$
\alpha_{2}^{*}=\pi / 2-\beta_{2}^{*} / 2, \quad \text { where } \quad \beta_{2}^{*}=2 \arcsin (1 /(1+r))
$$

are the angles in a tight triangle of type 2 , and

$$
\alpha_{3}^{*}=2 \arcsin (r /(1+r)) \quad \text { and } \quad \beta_{3}^{*}=\pi / 2-\alpha_{3}^{*} / 2
$$

are the angles in a tight triangle of type 3.

$$
\begin{aligned}
& \alpha_{0}=\arctan \left(\left((1+r) \sin \left(\alpha_{2}^{*}+\alpha_{3}^{*}\right)\right) /\left(2-(1+r) \cos \left(\alpha_{2}^{*}+\alpha_{3}^{*}\right)\right)\right) \quad \text { and } \\
& \beta_{0}=\pi-\left(\alpha_{0}+\alpha_{2}^{*}+\alpha_{3}^{*}\right)
\end{aligned}
$$

are the other two angles of a $T[1,1, r]$ triangle in which an angle of size $\alpha_{2}^{*}+\alpha_{3}^{*}$ is enclosed by two sides of length 2 and $(1+r)$, respectively.

The numerical values are given in Table 2 .

Table 2. Some characteristic data of the target packings.

\begin{tabular}{rcccccc}
\hline & $\mathcal{P}_{1}$ & $\mathcal{P}_{2}$ & $\mathcal{P}_{3}$ & $\mathcal{P}_{4}$ & $\mathcal{P}_{5}$ & $\mathcal{P}_{6}$ \\
\hline$r=$ & $0.63755 \ldots$ & $0.53329 \ldots$ & $0.41421 \ldots$ & $0.34919 \ldots$ & $0.28077 \ldots$ & $0.15470 \ldots$ \\
$\alpha_{2}^{*}=$ & $0.91389 \ldots$ & $0.86032 \ldots$ & $0.78539 \ldots$ & $0.73596 \ldots$ & $0.67488 \ldots$ & $0.52359 \ldots$ \\
$\beta_{2}^{*}=$ & $1.31380 \ldots$ & $1.42093 \ldots$ & $1.57079 \ldots$ & $1.66965 \ldots$ & $1.79181 \ldots$ & $2.09439 \ldots$ \\
$\alpha_{3}^{*}=$ & $0.79981 \ldots$ & $0.71046 \ldots$ & $0.59450 \ldots$ & $0.52359 \ldots$ & $0.44203 \ldots$ & $0.26875 \ldots$ \\
$\beta_{3}^{*}=$ & $1.17088 \ldots$ & $1.21556 \ldots$ & $1.27354 \ldots$ & $1.30899 \ldots$ & $1.34977 \ldots$ & $1.43641 \ldots$ \\
$\alpha_{0}=$ & $0.62782 \ldots$ & $0.65407 \ldots$ & $0.67586 \ldots$ & $0.68044 \ldots$ & $0.67488 \ldots$ & $0.60489 \ldots$ \\
$\beta_{0}=$ & $0.80005 \ldots$ & $0.91672 \ldots$ & $1.08582 \ldots$ & $1.20158 \ldots$ & $1.34977 \ldots$ & $1.74433 \ldots$ \\
\hline
\end{tabular}




\section{Proof of the Theorem}

Recently the claim of the Theorem for $k=3$ was proved by the author in [He]. The method used there was a precursor of the present one, however, it exploited the fact that in $\mathcal{P}_{3}$ all tight triangles are congruent, so this method could not be used without essential extension in cases $k=1,2,4$ and 5, when this property does not hold.

In many parts of the proof, when the argument is simultaneously valid in all cases, i.e. for all considered values of $k$, we use simplified notation omitting the subscript or argument $k$. For the sake of completeness case $k=3$ is included.

The major steps of our proof are the following:

Step 1. In Section 3.1 the definition of the surplus area $a^{+}$(a measure of efficiency of the packing in a domain) is introduced and some characteristic data of the target packings are tabulated.

Step 2. In Section 3.2 lower bounds are given for the surplus area in a $T_{2}$ triangle-in the form of quasi-concave step functions in terms of an angle of the triangle.

Step 3. In Section 3.3, based on Step 2, lower bounds are derived for the surplus area in feasible $T_{1}$ triangles - in terms of an angle of the triangle.

Step 4. In Section 3.4 it is shown that triangles of large support circles can be densely packed only if the density is small in a neighboring triangle.

Step 5. In Section 3.5 a flexible cell building method is described and applied to give an actual decomposition rule, which depends on the surplus area and the type of triangle.

Step 6. Section 3.6 is devoted to showing that the surplus area cannot be negative in any of the cells, and concludes the proof of the Theorem.

\subsection{Step 1. The Surplus Area Function}

The surplus area function (introduced in [He]) is a key notion in the investigations. Consider a saturated packing of admissible discs, its FM-triangulation and a density value $\delta^{*}$. Let $a$ denote the area of the triangle and $s$ the sector-sum of it, i.e. the sum of the areas $s_{i}, i=1,2,3$, of the three disc sectors lying in the triangle. The surplus area $a^{+}$of the triangle (with reference to the target density $\delta^{*}$ ) is defined as

$$
a^{+}=a-s / \delta^{*} .
$$

It measures the (usually positive) area that can be "borrowed" from the triangle—and added to others-so that the density will not exceed $\delta^{*}$ in the rest of the triangle. The sign of this function indicates whether the (original) density is larger than $\delta^{*}$ or not, and it vanishes if the packing density is just $\delta^{*}$.

To keep this property valid we define the surplus area on the union of disjoint triangles as the sum of the surplus areas of the triangles. Thus we get a simple function which is additive on disjoint polygonal domains. It is more conveniently used than the density if domains of different density values have to be added. From our point of view, the surplus area of a domain carries more information than the density does. While the surplus area 
of the union of two disjoint domains is simply the sum of the two relevant values, it is not enough to know the densities in the parts to calculate the density in the union.

A domain is said to be positive (negative) if its surplus area is positive (negative).

\subsection{Step 2. Surplus Area Lower Bounds in $T_{2}$ Triangles}

Our next goal is to give simple lower bounds for the surplus area of feasible triangles of a given type in terms of an angle, typically the angle at the center of a small disc. Seeking a lower bound for a given class of triangles with all angles fixed we can assume that at least two discs are in contact (i.e. the triangle belongs to type $T_{1}$ ), since contraction of a triangle makes its support circle shrink, keeps its angles and reduces its area. First we study the behavior of this function in $T_{2}$ triangles to lay the foundation for developing lower bounds for the surplus area in the different $T_{1}$ subclasses.

3.2.1. The Shape of the Surplus Area Function. In each of the $T_{2}$ subclasses two sides of the triangle are of a given length so the triangle is fully determined by its "middle angle," the angle enclosed by these sides. Keeping in mind that the radius of the support circle must be smaller than $r$ it is easy to determine the greatest lower and smallest upper bounds limiting the size of the middle angle in feasible $T_{2}$ triangles. The lower bound of this angle (attained for a tight $T_{3}$ triangle) belongs to the feasible interval, and the upper bound does not, because that would admit a support circle of radius $r$.

The feasibility intervals of the middle angle for the $T_{2}$ subclasses are listed in Table 3 .

For the surplus area function of a $T_{2}$ triangle the following lemma holds.

Lemma 1. In every subclass $T_{2}$ the sector-sum $s$ is a concave function of the middle angle.

Proof. In the "symmetric" cases, i.e. when the first and third arguments of $T_{2}$ are the same, the function is even linear.

Because of the similarity of the two remaining cases $T_{2}[1,1, r]$ and $T_{2}[1, r, r]$ we discuss only case $T_{2}[1,1, r]$.

Let $A_{1}, A_{2}$ and $B$ be the vertices of the triangle and let $\alpha_{1}, \alpha_{2}$ and $\beta$ be the opposite angles (Fig. 10). Suppose that $\alpha_{1}$ is the middle angle and that $\alpha_{1}=\alpha_{0}-\delta, \alpha_{1}=\alpha_{0}$ and $\alpha_{1}=\alpha_{0}+\delta$ are also feasible angles belonging the positions $A_{2}^{\prime}, A_{2}$ and $A_{2}^{\prime \prime}$ of the

Table 3. Feasibility intervals of the middle angle in $T_{2}$ triangles.

\begin{tabular}{ll}
\hline$T_{2}[1,1,1]:$ & $\alpha_{1} \in\left[\pi / 3,2 \alpha_{2}^{*}\right)$ \\
$T_{2}[1,1, r]:$ & $\alpha_{1} \in\left[\alpha_{2}^{*}, \alpha_{2}^{*}+\alpha_{3}^{*}\right)$ \\
$T_{2}[1, r, 1]:$ & $\beta \in\left[\beta_{2}^{*}, 2 \beta_{3}^{*}\right)$ \\
$T_{2}[1, r, r]:$ & $\beta_{1} \in\left[\beta_{3}^{*}, \beta_{3}^{*}+\pi / 3\right)$ \\
$T_{2}[r, 1, r]:$ & $\alpha \in\left[\alpha_{3}^{*}, 2 \alpha_{3}^{*}\right)$ \\
$T_{2}[r, r, r]:$ & $\beta_{1} \in[\pi / 3,2 \pi / 3)$ \\
\hline
\end{tabular}




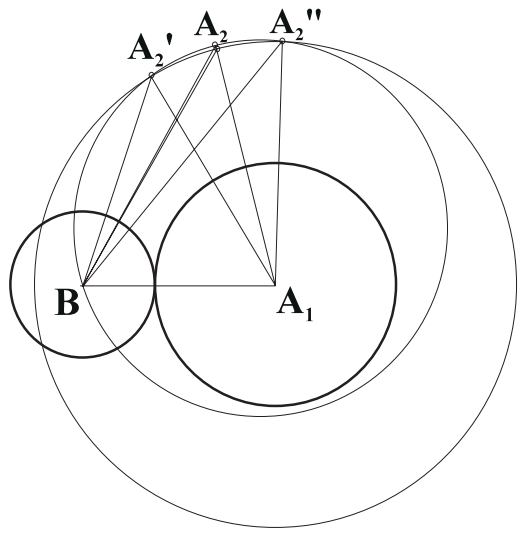

Fig. 10

other vertex marked 1. $A_{2}$ is lying half way between $A_{2}^{\prime}$ and $A_{2}^{\prime \prime}$ on the circle of radius 2 about $A_{1}$ and it is inside the circle $A_{2}^{\prime} A_{2}^{\prime \prime} B$, consequently, the value of $\beta$ belonging to $A_{2}$ is smaller than the average of its values belonging to positions $A_{2}^{\prime}$ and $A_{2}^{\prime \prime}$. Then the sector-sum at $A_{2}$ is larger than its average.

Lemma 1 (and its simple corollaries) help us to show that, in every subclass of $T_{2}$ triangles, to find the largest constant lower bound of $a^{+}$over a given interval $I$ of the middle angle, it will be enough to calculate its value at the endpoints of $I$.

It is worth noting that while for any fixed $r<1$ the absolute maximum of the packing density in a feasible $T_{2}$ triangle is attained for the tight triangle $T_{3}[1, r, r]$ (see [F11]), the surplus area may behave differently. In our cases, however, the surplus area of a feasible triangle, as we shall see, is minimal for $T_{3}[1, r, r]$.

For each $k$, the value of the surplus area in the tight triangle $T_{3}[1, r, r]$ will be denoted by $-v$. Necessarily, $v$ is positive, since $\delta^{*}$ is the density of an existing tiling, while the density in $T_{3}[1, r, r]$ provides an unattainable upper bound. The surplus area of the tight triangle of type $i$ will be denoted by $v_{i}, i=1, \ldots, 4$. To simplify some forms we also use the notation $v_{5}=\left(v_{3}+v_{4}\right) / 2$.

Remark. Let $x_{1}<x_{2}<x_{3}<x_{4}<x_{5}$ and let $f(x)$ be a quasi-concave function in interval $\left[x_{1}, x_{5}\right]$. Clearly, if $f\left(x_{2}\right)<f\left(x_{3}\right)$ and $f\left(x_{4}\right)<f\left(x_{3}\right)$, then $f(x)<f\left(x_{2}\right)$ for $x \in\left[x_{1}, x_{2}\right)$ and $f(x)<f\left(x_{4}\right)$ for $x \in\left(x_{4}, x_{5}\right]$. Due to this property of the quasi-concave functions the numerical calculations necessary to develop the required bounds on the surplus area function could be reduced to a few substitutions into simple trigonometric functions.

Now, for later use, we introduce a constant $\mu>6 v$. The values of $\nu, v_{i}$ and $\mu$ are given in Table 4.

Data gained from numerical calculations suggest that the surplus area is a quasiconcave function of any $\beta$-angle, however, in view of the proof of the Theorem it will be sufficient to prove a weaker statement formulated in the following lemma. 
Table 4. Surplus area in tight triangles.

\begin{tabular}{lllllll}
\hline & \multicolumn{1}{c}{$\mathcal{P}_{1}$} & \multicolumn{1}{c}{$\mathcal{P}_{2}$} & \multicolumn{1}{c}{$\mathcal{P}_{3}$} & \multicolumn{1}{c}{$\mathcal{P}_{4}$} & \multicolumn{1}{c}{$\mathcal{P}_{5}$} & \multicolumn{1}{c}{$\mathcal{P}_{6}$} \\
\hline$v$ & $0.00009243 \ldots$ & $0.0001011 \ldots$ & $0.0004166 \ldots$ & $0.0006757 \ldots$ & $0.0004916 \ldots$ & $0.0005572 \ldots$ \\
$\nu_{1}$ & $>70 v$ & $>130 v$ & $>50 v$ & $>40 v$ & $>90 v$ & $>120 v$ \\
$v_{2}=v / 3$ & $=2 v$ & $=0$ & $>-v / 2$ & $=v / 2$ & $=0$ \\
$v_{3}=-v$ & $=-v$ & $=-v 1$ & $=-v$ & $=-v$ & $=-v$ \\
$v_{4}$ & $>31 v$ & $>37 v$ & $>10 v$ & $=6 v$ & $>7 v$ & $>3 v$ \\
$v_{5}$ & $>15 v$ & $>18 v$ & $>4.5 v$ & $=2.5 v$ & $>3 v$ & $>v$ \\
$\mu$ & 0.00056 & 0.00061 & 0.0025 & 0.0041 & 0.003 & 0.00335 \\
\hline
\end{tabular}

Lemma 2. In each subclass the surplus area is larger than $\mu$ in a single subinterval of the feasible range of the middle angle and of any $\beta$-angle. $a^{+}<\mu$ is possible only in subintervals adjacent to the endpoints of the feasibility interval.

Proof. Symmetric subclasses. The surplus area $a^{+}=a-s / \delta^{*}$ is trivially a concave function of the middle angle of a $T_{2}$ triangle in the four symmetric subclasses, i.e. when the two discs touching the middle one are congruent and, consequently, the sector-sum $s$ is a linear function of the middle angle. In these cases it is easy to find the required subinterval of the middle angle.

Since the size of any $\beta$-angle different from the middle angle is a strictly decreasing function of the middle angle in a symmetric subclass, condition $a^{+} \geq \mu$ defines a single interval of the chosen $\beta$-angle even when it is not the middle angle.

Asymmetric subclasses. (See Fig. 11.) By Lemma $1 s$ is a concave (actually very flat) function of the middle angle, and it can be replaced by simple upper bounds in subclasses $T_{2}[1,1, r]$ and $T_{2}[1, r, r]$ when $s$ is not linear itself.

In subclass $T_{2}[1,1, r]$ the linear function of the middle angle $\alpha_{1}$, based on the lower endpoint tangent of the concave function $s\left(\alpha_{1}\right)$, provides an upper bound $l\left(\alpha_{1}\right) \geq s\left(\alpha_{1}\right)$. Let $\delta>0$ be the smallest value such that $\mu=a-l\left(\alpha_{2}^{*}+\delta\right) / \delta^{*} \leq a^{+}$. In fact, for all $k$, this concave lower bound of $a^{+}$is larger than $\mu$ at the other end of the feasibility interval. Then we can conclude that $a^{+}>v_{2}$ in $\alpha_{1} \in\left[\alpha_{2}^{*}, \alpha_{2}^{*}+\delta\right)$ and $a^{+}>\mu$ holds after that. Here again $\beta$ is a strictly decreasing function of the middle angle, thus those intervals

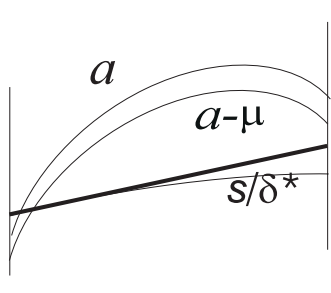

(a)

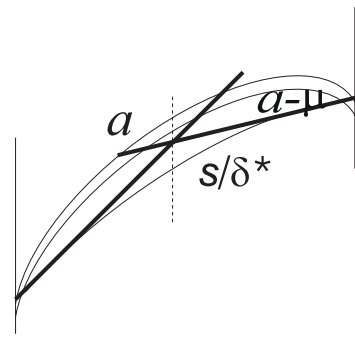

(b)

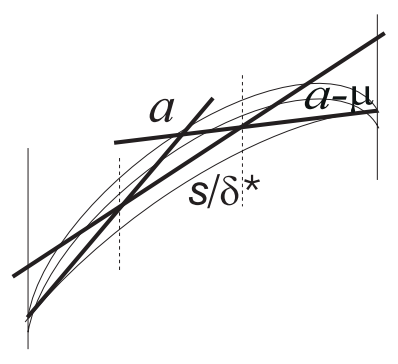

(c)

Fig. 11 
of the middle angle where the different lower bounds hold are mapped (monotonously) onto intervals of $\beta$.

Similarly, in subclass $T_{2}[1, r, r]$ the minimum $l\left(\beta_{1}\right)$ of the two linear functions, $l_{1}\left(\beta_{1}\right)$ and $l_{2}\left(\beta_{1}\right)$, the endpoint tangents, give an upper bound for $s$. For $k<6$ this implies

$a^{+}>\mu$ if $\beta_{1} \in\left(\beta_{3}^{*}+\varepsilon, \beta_{3}^{*}+\pi / 3-\varepsilon^{\prime}\right)$ for some non-negative values $\varepsilon$ and $\varepsilon^{\prime}$,

$a^{+} \geq v_{3}$ (the value of $a^{+}$at $\beta_{1}=\beta_{3}^{*}$ ) if $\beta_{1} \in\left(\beta_{3}^{*}, \beta_{3}^{*}+\varepsilon\right)$ and

$a^{+} \geq v_{5}$ (the value of $a^{+}$at $\beta_{1}=\beta_{3}^{*}+\pi / 3$ ) if $\beta_{1} \in\left(\beta_{3}^{*}+\pi / 3-\varepsilon^{\prime}, \beta_{3}^{*}+\pi / 3\right.$ ).

Unfortunately, in the case $k=6$ the upper bound of sector-sum $s$, based on the endpoint tangents, is not good enough and, to guarantee that the surplus area is uninterruptedly larger than $\mu$, a third linear cut is used in the midsection of the feasibility interval. The details of this (otherwise simple) calculation (illustrated in Fig. 11(c)) are not presented here. Again the other $\beta$ is a strictly decreasing function of the middle angle and the intervals where the different lower bounds hold can be mapped onto intervals of (the other) $\beta$.

3.2.2. Calculated Lower Bounds in $T_{2}$ Triangles. Next, intervals will be calculated for the lower bounds of the surplus area in terms of each $\beta$-angle to show that triangles of high density are "almost tight" or "almost stretched."

In every $T_{2}$ triangle the $\beta$-angle(s), if different from the middle angle, can be easily calculated from the middle angle. Due to Lemma 2 any pair of $\beta$-values, where $\alpha^{+}>\mu$, determines such an interval. (The numerical values are rounded and do not always define exactly the same intervals for the different angles, but the direction of the rounding ensures that all lower bounds remain valid.)

In $T_{2}[1,1, r]$ triangles of middle angle $\alpha_{1}$ the following formulas are used:

$$
\begin{aligned}
\beta & =\pi-\left(\alpha_{1}+\alpha_{2}\right), \\
\alpha_{2} & =\arctan \left((1+r) \sin \alpha_{1} /\left(2-(1+r) \cos \alpha_{1}\right)\right),
\end{aligned}
$$

and in $T_{2}[1, r, r]$ triangles of middle angle $\beta_{1}$,

$$
\begin{aligned}
\beta_{2} & =\pi-\left(\beta_{1}+\alpha\right), \\
\alpha & =\arctan \left(2 r \sin \beta_{1} /\left(1+r-2 r \cos \beta_{1}\right)\right) .
\end{aligned}
$$

The bounds are summarized in Table 5 .

Observe the fact that the endpoints of these subintervals are fairly close to the endpoints of the feasible intervals in each case, hence even a small deviation in any $\beta$-angle from the corresponding "best" triangles results in a surplus area larger than $\mu$.

Notice that the surplus area of a type 1 feasible triangle is unconditionally larger than $\mu$. The same holds for type 4 triangles, however, only for $k<6$. In all other cases the feasible range of the angle at a vertex marked $r$ can be cut into two or three parts: one large subinterval where the surplus area is at least $\mu$ and one or two very small intervals 
Table 5. Lower bounds.

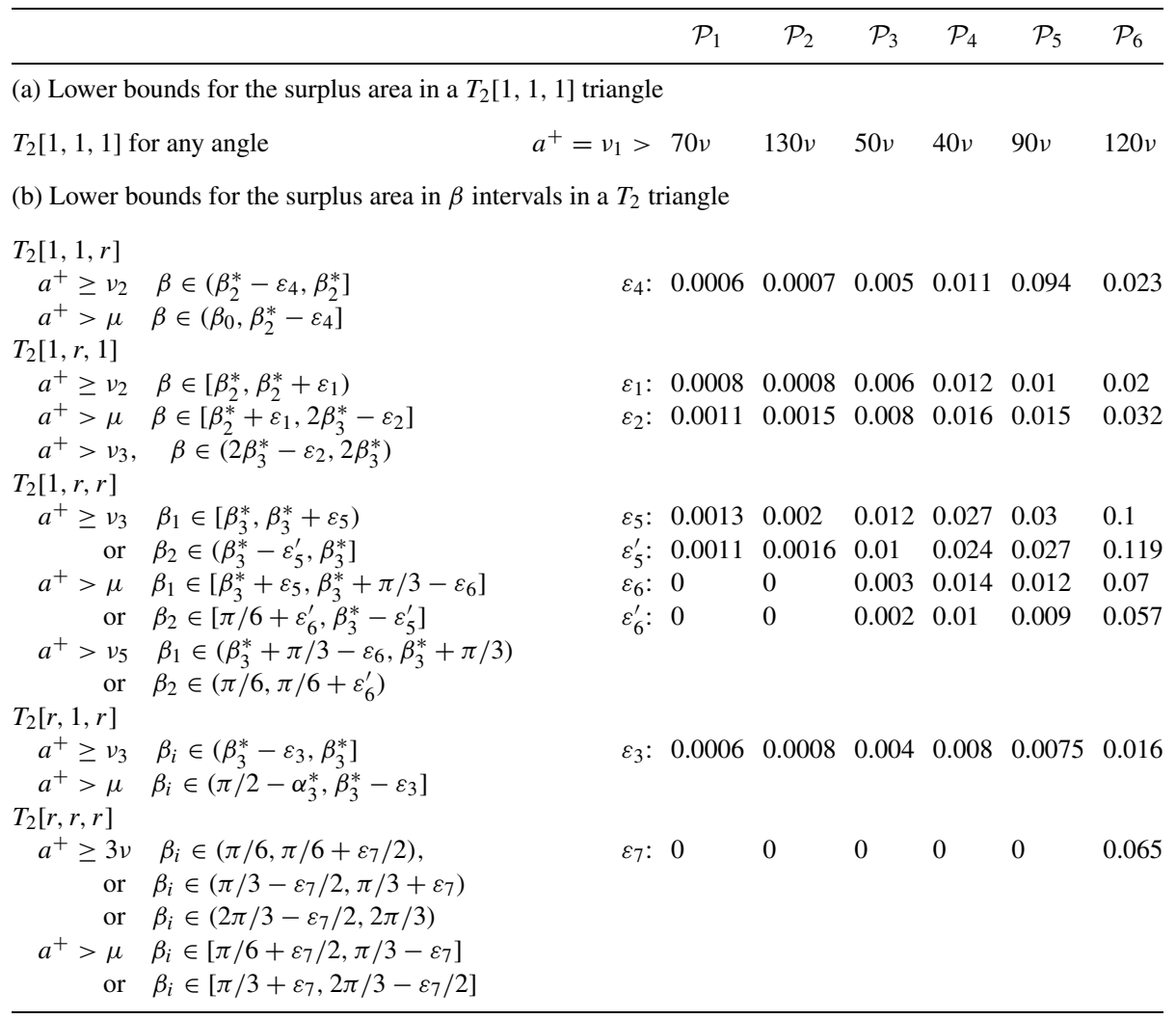

at one or both ends of the feasibility interval where only a weaker lower bound holds. In each case the inequality is strict except for the single value representing the tight triangle of the subclass.

For easier reference we give simpler notation to certain intervals, which play an important role in the rest of the proof:

$$
\begin{aligned}
I_{2,1} & \equiv\left[\beta_{2}^{*}-\varepsilon_{4}, \beta_{2}^{*}+\varepsilon_{1}\right], \\
I_{2,2} & \equiv\left[2 \beta_{3}^{*}-\varepsilon_{2}, 2 \beta_{3}^{*}\right], \\
I_{3,1} & \equiv\left[\beta_{3}^{*}-\varepsilon_{5}^{\prime}, \beta_{3}^{*}+\varepsilon_{5}\right], \\
I_{3,2} & \equiv\left[\pi / 6, \pi / 6+\varepsilon_{6}^{\prime}\right], \\
I_{3,3} & \equiv\left[\beta_{3}^{*}+\pi / 3-\varepsilon_{6}, \beta_{3}^{*}+\pi / 3\right], \\
I_{4,1} & \equiv\left[\pi / 3-\varepsilon_{7} / 2, \pi / 3+\varepsilon_{7}\right], \\
I_{4,2} & \equiv\left[\pi / 6, \pi / 6+\varepsilon_{7} / 2\right], \\
I_{4,3} & \equiv\left[2 \pi / 3-\varepsilon_{7} / 2,2 \pi / 3\right] .
\end{aligned}
$$


Using this notation the results of this section can be summarized in:

Corollary 1. If the surplus area of a $T_{2}$ triangle $T$ is smaller than $\mu$, then one of the following cases holds:

(i) $T$ belongs to type 2 and either $\beta \in I_{2,1}$ or $\beta \in I_{2,2}$.

(ii) $T$ belongs to type 3 and $\beta_{i} \in I_{3,1}, i=1,2$.

(iii) $k>2, T$ belongs to type 3 and the smaller $\beta$-angle is $\in I_{3,2}$ and the larger one is $\in I_{3,3}$.

(iv) $k=6, T$ belongs to type 4 and $\beta_{i} \in I_{4,1}, i=1,2,3$, or the largest angle is $\in I_{4,3}$ and the two other angles are $\in I_{4,2}, i=1,2$.

\subsection{Step 3. Surplus Area Lower Bounds in $T_{1}$ Triangles}

Consider a saturated packing of unit discs and discs of radius $r<1$, a triangle $A B C$ of its marked FM-triangulation and a target density denoted by $\delta^{*}$. Assume further that $\delta^{*}>1 /(1+r)^{2}$, which is valid in the cases covered by the Theorem. Let us move vertex $C$ on the straight line $A C$ increasing the angle $\angle A B C$. The following lemma holds:

Lemma 3. For given side $A B$ and given angle $\angle B A C$ the surplus area of $A B C$ is an increasing function of the angle $\angle A B C$.

Proof. The claim is trivially true if the disc around $C$ is a unit disc or the disc around $B$ and $C$ are congruent, since, by the move, the area of the triangle increases and the sectorsum $s$ does not. Assume this is not the case: i.e. $B$ is marked 1 and $C$ is marked $r$. The area of the triangle increases with slope $(1+r)^{2} / 2$ and the sum of the sectors increases with slope $\left(1-r^{2}\right) /\left(2 \delta^{*}\right)$. Thus the slope of the surplus area function is positive.

Corollary 2. Suppose that the disc around $C$ does not touch the other discs. Then vertex $C$ can be moved toward $A$ on side $A C$ (together with the disc around it) to a position $C^{\prime}$ in which the disc hits one of the other discs without increasing the radius of the support circle and during this move the surplus area of the triangle decreases.

Proof. Let $P$ be the center of the support circle. Clearly, $A P<A C$, which implies that during the move the radius of the support circle increases with $\angle A B C$. Consequently, moving $C$ in the opposite direction, toward $A$, will reduce the radius of the support circle and will not cause infeasibility.

In the rest of this section we investigate the different $T_{1}$ subclasses of feasible triangles and develop step function lower bounds for the surplus area, especially, intervals of the $\beta$-angles (or $\beta_{i}$-angles) will be determined in which the surplus area is larger than $\mu$.

In a $T_{1}\left[r_{1}, r_{2}, r_{3}\right]$ triangle the side of length $r_{1}+r_{2}$ connecting the vertices of discs in contact will be called the base and the vertex opposite the base will be called the apex. For a fixed base the locus of the apex is a domain bounded by three (circular) 
arcs according to the three feasibility conditions: the distance of the apex from the base vertices is bounded from below (by $r_{1}+r_{3}$ and $r_{2}+r_{3}$, respectively), and the condition on the size of the support circle gives an upper bound. This third boundary arc is limiting the distance of the apex from the center of a circle of radius $r$ touching the two discs around the base vertices. The interior points of the first two arcs and their common endpoint are feasible while the points of the third arc (which would admit a support circle of radius $r$ ) are not. In most cases the surplus area of the selected $T_{1}$ triangle will be compared with that of a $T_{2}$ triangle of the same $T_{1}$ class (having the same $\beta$-angle) and the bounds found in the previous section will be applied.

Starting with a $T_{1}$ triangle we select an angle of it (a $\beta$-angle, except in the simple case of $T_{1}[1,1,1]$, when only $\alpha$-angles exist) and consider the locus of the apex for which the selected angle is constant. The different cases are discussed in two groups depending on the position of the angle selected.

3.3.1. Lower Bounds Depending on an Angle at the Base. If the selected angle is at the base, then the locus of the apex is the intersection of the feasibility region and the half-line determining the angle in question. By Corollary 2 (of Lemma 3) the surplus area reduces if the apex is moved toward the selected vertex on the base. The endpoint of this line segment, nearest to the base, lies on the feasible boundary arc representing a $T_{2}$ triangle, since moving the apex reduces the size of the support circle, therefore this move cannot be blocked by the support circle condition, which is sharp for the infeasible boundary arc (see Fig. 12).

Consequently, for every subset of given base angle of a $T_{1}$ subclass the $T_{2}$ triangle (of it) has the smallest surplus area. Due to this fact the $T_{1}$ triangles of a given base angle inherit the lower bounds valid for the corresponding $T_{2}$ triangles. If the given angle is such that it fulfills the angle condition in the resulting $T_{2}$ triangle to have a surplus area $a^{+}>c$, then the same bound is valid for the original $T_{1}$ triangle as well.

3.3.2. Lower Bounds Depending on the Angle at the Apex. We are interested only in the cases when the given angle lies at a vertex marked $r$. These two cases are discussed separately:

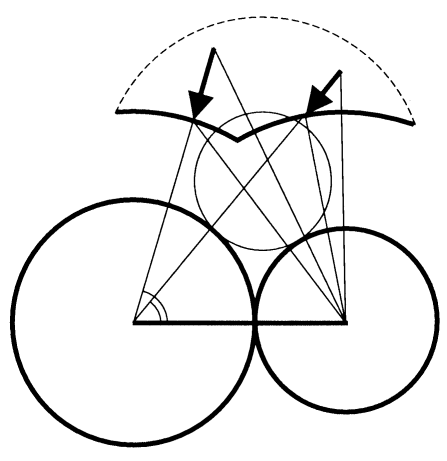

Fig. 12 




Fig. 13

3.3.2.1. Case 1: Base vertices marked 1 and 1 , apex marked $r$. Triangle $A_{1} A_{2} B \equiv$ $T \in T_{1}[1,1, r], A_{1} A_{2}=2$, apex angle $\beta$ is given.

Denote by $B^{\prime}$ the "top" of the feasibility region, the point representing the triangle of largest area, by $\left.\beta^{\prime}=2 \arctan \left(1 /\left(2 r+\tan \left(\sqrt{(} 2 r+r^{2}\right)\right)\right)\right)$ the angle $\angle A_{1} B^{\prime} A_{2}$ and by $C$ an endpoint of the infeasible boundary arc. (The angle $\angle A_{1} C A_{2}$ coincides with the angle $\beta_{0}$ defined in Section 2, Table 2).

In this case-with $\beta$ given - the sector-sum is constant, thus the triangle of minimal area is the triangle of minimal surplus area. Consider first a triangle whose apex $B$ lies inside the circle $A_{1} A_{2} C$ (and in the feasibility region). On one hand, moving $B$ on the circumcircle of the triangle nearer to the base decreases the area, on the other handconsidering the fact that $r<1$-the boundary point reached this way is lying on a feasible boundary arc, representing a $T_{2}$ triangle of the subclass (Fig. 13).

If $B$ lies in the other part of the feasibility region, then the apex angle is not represented among $T_{2}$ triangles, thus this subcase requires different treatment. In this subregion we give lower bounds for the area and upper bounds for the sector-sum. The area is clearly bounded from below by the area $a_{\min }=(1+r) \sin \left(\alpha_{2}^{*}+\alpha_{3}^{*}\right)$ of the triangle $A_{1} A_{2} C$ and $s$ attains its largest value $s_{\max }$ where $\beta$ is smallest, i.e. at $B^{\prime}$. The values given in Table 6 show that the surplus area of each triangle in this set exceeds $\mu$ definitely.

Since all $T_{1}$ triangles with $\beta \in\left(\beta^{\prime}, \beta_{0}\right]$, i.e. outside the feasibility range of its $T_{2}$ triangles, have a surplus area $>\mu$, we can say that the surplus area of a $T_{1}$ triangle of some $\beta$ angle can be $<\mu$ only if $\beta$ fulfills the necessary condition for the $T_{2}$ triangles of this $T_{1}$ class to have a surplus area $<\mu$.

Table 6. Surplus area lower bounds for the "upper" half of the feasibility region.

\begin{tabular}{llllllc}
\hline & $\mathcal{P}_{1}$ & \multicolumn{1}{c}{$\mathcal{P}_{2}$} & \multicolumn{1}{c}{$\mathcal{P}_{3}$} & \multicolumn{1}{c}{$\mathcal{P}_{4}$} & $\mathcal{P}_{5}$ & $\mathcal{P}_{6}$ \\
\hline$a_{\min }=$ & $1.620 \ldots$ & $1.533 \ldots$ & $1.388 \ldots$ & $1.284 \ldots$ & $1.151 \ldots$ & $0.822 \ldots$ \\
$s_{\max }=$ & $1.350 \ldots$ & $1.269 \ldots$ & $1.156 \ldots$ & $1.081 \ldots$ & $0.987 \ldots$ & $0.745 \ldots$ \\
$a_{\min }-s_{\max } / \delta^{*}=$ & $0.137 \ldots$ & $0.145 \ldots$ & $0.132 \ldots$ & $0.114 \ldots$ & $0.091 \ldots$ & $0.037 \ldots$ \\
$\mu=$ & 0.00056 & 0.00061 & 0.0025 & 0.0041 & 0.003 & 0.00335 \\
\hline
\end{tabular}





Fig. 14

Remark. This case shows that the feasible angle range of a $T_{1}$ subclass and that of its $T_{2}$ subset can be different.

3.3.2.2. Case 2: Base vertices marked 1 and $r$, apex marked $r$. Triangle $A B_{1} B_{2} \equiv$ $T \in T_{1}[1, r, r], A B_{1}=1+r$, apex angle $\beta_{2}$ is given. $C_{1}$ and $C_{2}$ are the endpoints of the infeasible boundary arc, $C_{1}$ closer to $B_{1}$ (Fig. 14).

Subcase 1: $\beta_{1} \geq \pi / 2$. We prove that (in this subcase) for any fixed feasible $\beta_{1}$ the minimum of the surplus area is attained for the $T_{2}[1, r, r]$ triangle of the same apex angle, thus the lower bounds valid for the latter in terms of $\beta_{2}$ are valid for the whole subset. Consider the locus (arc) of the apex for which the apex angle $\beta_{2}$ is the same and assume that $B_{2}$ does not lie on the feasible boundary arc. We first shift the small disc centered at $B_{1}$ toward the one about $B_{2}$ until contact, then we shift the unit disc until it hits the other small discs. The resulting triangle has the same apex angle and (at least) two contacts and thus it is congruent with the one represented by the common point of the locus and the feasible boundary arc. On the other hand, during the two steps of this operation the surplus area was reduced (Corollary 2). This settles the claim for Subcase 1.

Subcase 2: $\beta_{1} \leq \pi / 2$. For this subset the validity of the $T_{2}$ lower bounds will be extended again for the $T_{1}$ triangles by comparing each $T_{1}$ triangle with a $T_{2}[1, r, r]$ or $T_{2}[r, 1, r]$ triangle of the same class.

First the (reduced) feasibility domain will be cut further into two parts by the circle $L$ containing the apexes of angle $\beta_{2}=\beta_{3}^{*}-\varepsilon_{5}^{\prime}$. Denote the points of intersection of this circle and the boundary arcs representing the $T_{2}[1, r, r]$ and $T_{2}[r, 1, r]$ triangles by $R$ and $S$, respectively. Since (for all values of $k$ ) $\varepsilon_{3}<\varepsilon_{5}^{\prime}$ on one hand, and $a^{+} \geq \mu$ in the $T_{2}[1, r, r]$ triangle of $\beta_{1}=\pi / 2$ on the other hand, $a^{+} \geq \mu$ at both points $R$ and $S$ as well as on the subarcs $L_{1}$ and $L_{2}$ of both feasible boundary arcs of the reduced feasibility region outside circle $L$. Lower bound $a^{+} \geq \mu$ holds also on the entire arc $R S$ of $L$, since the sector-sum is a linear function and the triangle area is a concave function of the arc-length.

Consider now the case when apex $B_{2}$ is lying outside or on $L$ and connect it with vertex $A$ on the base. Let $P$ now be the point where segment $B_{2} A$ meets one of the arcs $L_{1}, L_{2}$ or $R S . P$ is the apex of a feasible $\left(T_{1}\right.$ or $\left.T_{2}\right)$ triangle. Comparison of the 
original triangle and the new one provides lower bound $\mu$ for the surplus area of the original triangle, since in this comparison the area of the second triangle is smaller and the sector-sum is the same in the two triangles.

If the apex $B_{2}$ is inside $L$, then the segment $B_{2} A$ leads to the apex of a $T_{2}$ triangle. The result of the comparison now leads to the general lower bound $a^{+} \geq v_{3}$ (the value for a tight triangle) for the surplus area of all $T_{1}$ triangles of the class. This settles Subcase 2 .

The results in Sections 3.3.1, 3.3.2.1 and 3.3.2.2 yield

\section{Lemma 4.}

(a) If $T$ is a triangle belonging to the $T_{1}$ class of type 1 , then $a^{+}>\mu$.

(b) Let $T$ be a triangle belonging to a $T_{1}$ class of type 2, type 3 or type 4 and let $\beta$ be an angle of it. If $\beta$ is such that this $T_{1}$ class contains a $T_{2}$ triangle with angle $\beta$, then the lower bounds given in Table 5(b) for $T_{2}$ triangles are valid for the surplus area of $T$. If it is not the case, then the surplus area of $T$ is larger than $\mu$.

It will be practical to give names to some kinds of triangles which have a small (large) surplus area. A triangle is fat if its surplus area is $\geq \mu$. A type 2 , type 3 or type 4 triangle is quasi-tight if its surplus area is $<\mu$ and its angles are "close" to those of a tight triangle, more exactly, if $\beta \in I_{2,1}, \beta_{i} \in I_{3,1}, i=1,2$, and $\beta_{i} \in I_{4,1}, i=1,2,3$, respectively. Similarly, a type 2 , type 3 or type 4 triangle is called quasi-stretched if its surplus area is $<\mu$ and its angles are "close" to those of a stretched triangle: for type 2 triangles $\beta_{i} \in I_{2,2}$, for the angles $\beta_{1} \leq \beta_{2}$ of type 3 triangles $(<\mu$ implies $k>2)$ $\beta_{1} \in I_{3,2}$ and $\beta_{2} \in I_{3,3}$, finally, for the angles $\beta_{1} \leq \beta_{2} \leq \beta_{3}$ of type 4 triangles $(<\mu$ implies $k=6) \beta_{1} \in I_{4,2}, \beta_{2} \in I_{4,2}, \beta_{3} \in I_{4,3}$. Since the intervals given in the above definitions do not overlap, quasi-tight and quasi-stretched triangles form disjoint sets.

The results of this section can be summarized in

Corollary 3. If the surplus area of a $T_{1}$ triangle $T$ is smaller than $\mu$, then one of the following cases holds:

(i) $T$ belongs to type 2 and either $\beta \in I_{2,1}$ or $\beta \in I_{2,2}$.

(ii) $T$ belongs to type 3 and $\beta_{i} \in I_{3,1}, i=1,2$.

(iii) $k>2$, T belongs to type 3 and its smaller $\beta$-angle is $\in I_{3,2}$ and the larger one is $\in I_{3,3}$.

(iv) $k=6, T$ belongs to type 4 , and $\beta \in I_{4,1}, i=1,2,3$, or the largest angle is $\in I_{4,3}$ and the two other angles are $\in I_{4,2}, i=1,2$.

\subsection{Step 4. Combined Lower Bound for Some Pairs of Adjacent Triangles}

In the following lemma we claim that some triangles of small surplus area and large support circle have an adjacent triangle of large surplus area.

Lemma 5. Let $T^{\prime}$ be a quasi-stretched triangle and let $T^{\prime \prime}$ be the neighboring triangle such that they share the longest side of $T^{\prime}$. Then $T^{\prime \prime}$ is fat. 
Sketch of Proof. Let us assume the contrary to the statement, that the surplus area of $T^{\prime \prime}$ is not larger than $\mu$. Then, by Corollary 3, it must be either quasi-tight or quasi-stretched, hence its type and shape is fairly limited. Observe that if $T^{\prime}$ is of type 2, type 3 or type 4, the endpoints of the common side are marked 1 and 1,1 and $r$, and $r$ and $r$, respectively. Accordingly, $T^{\prime \prime}$ can be of type 1 or type 2, type 2 or type 3 , and type 3 or type 4 .

Easily calculated lower bounds on the length of the largest side of the different quasi-stretched triangles and upper bounds on the length of all sides of quasi-tight and quasi-stretched triangles exclude all possibilities except the "mirror" arrangements, in which a type $i$ quasi-stretched $T^{\prime}$ triangle and a type $i$ quasi-stretched $T^{\prime \prime}$ triangle share their longest side.

In a second step, to exclude the "mirror" pairs, it can be shown—again based on simple estimations - that the center of the support circle of a quasi-stretched triangle is separated from the triangle by the line of the longest side. This, however, cannot be true for $T^{\prime}$ and $T^{\prime \prime}$ simultaneously.

\subsection{Step 5. Decomposition of the Plane into Balanced Cells}

3.5.1. The Corner Piece and the Balanced Cell. Turning back to the proof of the Theorem we define a decomposition of the plane.

Consider a packing and its marked FM-triangulation. Every triangle will be cut into three polygonal parts each containing a vertex of the triangle. In each triangle every vertex will be associated with a polygonal part containing it and with the disc sector centered at it. The corner piece is defined as the set of a polygonal part $p$ of area $a(p)$ and disc sector $s$ of area $a(s)$ lying in the same triangle and associated with the same vertex. The corner piece is marked with the marking of the vertex. We say that a corner piece marked $r$ is quasi-tight, quasi-stretched or fat if it is part of such a triangle. The corner piece inherits the type of the triangle as well. In quasi-stretched type 3 triangles (which exist for $k>2$ only) the corner pieces marked $r$ are further distinguished: a piece is called acute or obtuse if the angle of the associated disc sector is acute or obtuse, respectively.

(Notice that a part of the triangle is not required to contain the associated sector, although both are lying in the same triangle.)

The surplus area of a corner piece is defined as $a(p)-a(s) / \delta^{*}$ (see Section 3.1), thus the surplus area of a triangle is the sum of the surplus areas of its three corner pieces, and the additive property of this function is maintained.

By definition, the cell of a disc is the union of the corner pieces having a common vertex, the center of the disc. A disc does not necessarily lie completely in its cell, however, the disc and its cell are both covered by the union of the triangles meeting at its center, thus the diameter of the cell is bounded from above. (This property is required to extend the notion of density to the whole plane.)

Our goal is to prove that to each packing of discs of radius 1 and $r(k)$ there exists a decomposition of the triangles (of the FM-triangulation) generating a decomposition of the plane into cells (in the above sense), such that the surplus area in each cell is non-negative. To do this we need a triangle-splitting rule which produces balanced cells, i.e. cells with more or less equal density. In fact there are many ways to do this, since the 
definition of the corner pieces is very flexible. The rule of decomposition is defined so that, when applied to a target packing, it produces an equal (vanishing) surplus area in every cell, even if the cells of the target packing are of different structure $(k=1,2,4,5)$. We direct the reader's attention to the fact that the actual shape of the corner pieces does not play any role and (within reasonable limits) only the angles and the areas count. It can be easily checked that the area which will be associated to a corner piece to get the prescribed surplus area is always positive. (Even the smallest sector has an area larger than $v$.) This implies that the corner pieces can be realized by actually cutting the triangle into non-empty polygonal pieces. By choosing the rules below we intended to exploit the method's flexibility to simplify the proof of the Theorem as much as possible.

3.5.2. Setting the Rules to Create the Corner Pieces. In splitting the triangles into polygonal parts the areas of the parts are defined through the required surplus area. The following rules are used:

Rule 1. In a type 1 triangle the three corner pieces get an equal surplus area.

Since all type 1 triangles are fat, in each piece $a^{+} \geq \mu / 3 \geq 2 v$.

Rule 2. In type 2 and type 3 triangles all corner pieces marked 1 have a 0 surplus area.

Consequently, in type 2 triangles the surplus area of the single corner piece marked $r$ will be $a^{+} \geq v_{2}, a^{+} \geq-v$ and $a^{+} \geq \mu \geq 6 v$, if it is quasi-tight, quasi-stretched and fat, respectively, and in type 3 triangles the surplus area of the triangle will be shared by the corner pieces marked $r$ as follows:

Rule 3. In type 3 quasi-tight and fat triangles the surplus area of the corner pieces marked $r$ will be the same: $a^{+} \geq-v / 2$ and $a^{+} \geq \mu / 2 \geq 3 v$, respectively.

Rule 4. In type 3 quasi-stretched triangles the surplus area of the obtuse corner piece will be $2 v$, while for the acute piece we have $a^{+} \geq v_{5}-2 v$.

Rule 5. In type 4 triangles the surplus are is distributed evenly among the three pieces.

The type 4 triangles are fat for $k<6$, so-by Rule 5-we have $a^{+} \geq \mu / 3 \geq 2 v$. Therefore $a^{+} \geq v$ for $k=6$ holds.

For the reader's convenience the lower bounds are given in tabular form as well (see Table 7).

\subsection{Step 6. Surplus Area in the Resulted Balanced Cells}

3.6.1. Surplus Area in the Cell of a Unit Disc. Rules 1 and 2 immediately imply that the cell of a unit disc has a non-negative surplus area.

Consequently, we may turn our attention to cells of a small disc. 
Table 7. Surplus area lower bounds in corner pieces marked $r$.

\begin{tabular}{llllllll}
\hline & & $\mathcal{P}_{1}$ & $\mathcal{P}_{2}$ & $\mathcal{P}_{3}$ & $\mathcal{P}_{4}$ & $\mathcal{P}_{5}$ & $\mathcal{P}_{6}$ \\
\hline Type 2 corner pieces & & & & & & & \\
Quasi-tight & $\geq$ & $v / 3$ & $2 v$ & 0 & $-v / 2$ & $v / 2$ & 0 \\
Quasi-stretched & $\geq$ & $-v$ & $-v$ & $-v$ & $-v$ & $-v$ & $-v$ \\
Fat & $\geq$ & $6 v$ & $6 v$ & $6 v$ & $6 v$ & $6 v$ & $6 v$ \\
Type 3 corner pieces & & & & & & & \\
$\quad$ Quasi-tight & $\geq$ & $-v / 2$ & $-v / 2$ & $-v / 2$ & $-v / 2$ & $-v / 2$ & $-v / 2$ \\
Quasi-stretched, acute & $\geq$ & & & $5 v / 2$ & $v / 2$ & $v$ & $-v$ \\
Quasi-stretched, obtuse & $=$ & & & $2 v$ & $2 v$ & $2 v$ & $2 v$ \\
Fat & $\geq$ & $3 v$ & $3 v$ & $3 v$ & $3 v$ & $3 v$ & $3 v$ \\
Type 4 corner pieces & $\geq$ & $2 v$ & $2 v$ & $2 v$ & $2 v$ & $2 v$ & $v$ \\
\hline
\end{tabular}

3.6.2. Surplus Area in the Cell of a Small Disc. Suppose that there exists a small disc such that the surplus area of its cell is negative. It will be proved that this is not possible. First we show that the assumed negative cell must consist of certain kinds of corner pieces. Then the possible integer combinations for which the angles of these pieces can add up to $2 \pi$ will be determined. Finally, it will be checked that none of these combinations can have a negative surplus area.

Let the notation $I_{p} \equiv\left(2 \beta_{3}^{*}-2 \varepsilon_{5}^{\prime}, 2 \beta_{3}^{*}+2 \varepsilon_{5}\right]$ be introduced for the "double" of interval $I_{3,1}$.

Proposition 1. The total surplus area of all type 3 quasi-stretched acute pieces and the pieces lying in the longest-side-neighbors of their triangles is $\geq 2 v$.

Proof. By Lemma 5 a type 3 quasi-stretched acute piece-which exists only for $k>2$ and can be negative only for $k=4$-has a fat neighboring piece, part of a longestside-neighbor triangle, which either belongs to type 2 with $a^{+} \geq 6 v$ or to type 3 with $a^{+} \geq 3 v$. Although one such fat neighbor might be counted twice if it is of type 2 , the surplus area of the union of the type 3 quasi-stretched acute pieces and their assigned fat neighbors is $\geq 2 v$, if at least one exists.

Proposition 2. The total surplus area of the type 2 pieces and the type 3 quasi-tight pieces is $\geq-2 v$.

Proof. A type 2 quasi-stretched piece can be negative $(\geq-v)$ only if its angle lies in interval $I_{2,2}$, which is, with respect to $\varepsilon_{2} \leq 2 \varepsilon_{5}^{\prime}$, a subset of $I_{p}$. There can be at most two such pieces.

A type 3 quasi-tight piece can also be negative. Since the smallest angle of a type 3 piece is at least $\pi / 6$ the total angle of any five type 3 quasi-tight pieces is at most $11 \pi / 6$, consequently, the number of type 3 quasi-tight pieces (the type 3 pieces which can have negative surplus area) is at most four and their combined surplus area is $\geq-2 v$. The angle of each negative piece belongs to interval $I_{3,1}$, the "half" of $I_{p}$.

It can be easily seen that the total surplus area cannot be more negative than $-2 v$ even if a combination of type 2 quasi-stretched pieces and type 3 quasi-tight pieces is used. 
(Each type 2 quasi-stretched piece can be substituted by two type 3 quasi-tight pieces of the same total angle and the same lower bound for the total surplus area.)

For $k \neq 4$ no other pieces could generate a negative total surplus area (see Table 7), i.e. no subset of these pieces is more negative than $-2 v$ and the claim of Proposition 2 is proved.

For $k=4$ the situation is similar, however, a type 2 quasi-tight piece (of interval $I_{2,1}$ ) must also be considered, since now it has a negative lower bound $(\geq-v / 2)$. Nonetheless, the angle of such a piece is larger than that in $I_{3,1}$, therefore the same argument and bound $(\geq-2 v)$ hold for the total angle and total surplus area of all type 3 quasi-tight and all type 2 negative pieces.

Proposition 3. A negative cell consists of quasi-tight type 2 pieces of angle $\in I_{2,1}$, quasi-stretched type 2 pieces of angle $\in I_{p}$ and pairs of quasi-tight type 3 pieces, each pair of total angle $\in I_{p}$.

Proof. The lower bounds in Propositions 1 and 2 exclude, for every $k$, the use of type 3 quasi-stretched (acute or obtuse) pieces and all fat pieces in general. Then-in a negative cell-all type 3 pieces must be quasi-tight with angles belonging to interval $I_{3,1}$. The marking conditions imply that the number of type 3 pieces is even. (These pieces form adjacent pairs or pairs framing the sequence of one or two type 4 pieces at both ends.) Hence, the type 3 quasi-tight pieces can be considered to form pairs of total angle $\in I_{p}$.

Type 4 corner pieces also disqualify themselves for $k<6$ having a too large $(\geq 2 v)$ surplus area. Next, let $k=6$, where the lower bound is smaller. A type 4 piece is at least $v$, therefore not more than one of them can occur in a negative cell (as the total surplus area of the other pieces is $\geq-2 v$ ). If the surplus area of such a piece is smaller than $2 v$, then that of its carrying triangle is smaller than $6 v(\leq \mu)$, therefore its central angle lies in one of the following three intervals:

(i) $I_{4,2} \equiv\left[\pi / 6, \pi / 6+\varepsilon_{7} / 2\right]$,

(ii) $I_{4,1} \equiv\left[\pi / 3-\varepsilon_{7} / 2, \pi / 3+\varepsilon_{7}\right]$,

(iii) $I_{4,3} \equiv\left[2 \pi / 3-\varepsilon_{7} / 2,2 \pi / 3\right]$.

Case (i) can be excluded, since then the piece is a quasi-stretched acute piece having a fat neighbor, which would make the cell non-negative. Case (iii) is impossible, since even the smallest angle would leave room only for a single angle taken from $I_{p}$ (of surplus area $\geq-v$ ), thus it cannot lead to a negative cell.

It is easy to check that there exist no non-negative integers $p$ and $q$ such that the sum of $p$ angles from $I_{p}, q$ angles from $I_{2,1}$ and one angle from interval $I_{4,1}$ equals $2 \pi$, except when $p=1$ and $q=1$, however, for this combination-i.e. for one pair of quasi-tight type 3 pieces (or a single quasi-stretched type 2 piece), one quasi-tight type 2 piece and one quasi-tight type 4 piece - the total surplus area is non-negative (see Table 7).

Having excluded all angles but those of intervals $I_{2,1}$ and $I_{p}$, the next step is to determine the feasible combinations of them. A feasible combination means finding integers $p \geq 0$ and $q \geq 0$, such that $p$ angles can be taken from the interval $I_{p}$, and $q$ from interval $I_{2,1}$ such that their sum is $2 \pi$. 
Proposition 4. There exists at most one feasible combination for each $k$.

Proof. The required solution exists if and only if a (non-negative) integer combination of the lower ends of intervals $I_{2,1}$ and $I_{p}$ is at most $2 \pi$, while the same integer combination of the upper ends is at least $2 \pi$. Clearly, there are just a few cases to check.

The calculation shows that only the following combinations are feasible:

$$
\begin{aligned}
& \text { for } k=1, \quad p=1 \text { and } q=3 \text {, } \\
& \text { for } k=2, \quad p=2 \text { and } q=1 \text {, } \\
& \text { for } k=3, \quad p=0 \text { and } q=4 \text {, } \\
& \text { for } k=5, \quad p=1 \text { and } q=2 \text {, } \\
& \text { for } k=6, \quad p=0 \text { and } q=3 \text {. }
\end{aligned}
$$

There exists no feasible combination to yield $2 \pi$ for $k=4$. (The reason is that the target configuration does not consist merely of tight triangles of types 2 and 3.)

Proposition 4 implies that only these combinations might lead to a negative balanced cell. The lower bounds, however, based on Table 7, are zero for each feasible solution showing that none of these combinations admits a packing denser than the corresponding target packing.

This concludes the proof of the Theorem.

\section{References}

[B1] G. Blind, Ebene Lagerungen von Kreisen, deren Radien nicht sehr verschieden sind, Ph.D. Thesis, Technische Hochschule, Stuttgart, 1966.

[Bo] K. Böröczky, Oral communication.

[FeG] G. Fejes Tóth, Covering the plane with convex discs, Acta Math. Acad. Sci Hungar. 23(1-2) (1972), 263-270.

[FeL1] L. Fejes Tóth, Lagerungen in der Ebene auf der Kugel und im Raum, Springer-Verlag, Berlin, 1953; 2nd edn., 1972.

[FeL2] L. Fejes Tóth, Regular Figures, Pergamon Press, Oxford, 1964.

[FeL3] L. Fejes Tóth, Compact packing of circles, Studia Sci. Math. Hungar. 19 (1984), 103-107.

[F11] A. Florian, Ausfüllung der Ebene durch Kreise, Rend. Circ. Mat. Palermo 9(2) (1960), 1-13.

[F12] A. Florian, Dichteste Packung inkongruenter Kreise, Monatsh. Math. 67(3) (1963), 229-242.

[FM] L. Fejes Tóth and J. Molnár, Unterdeckung und Überdeckung der Ebene durch Kreise, Math. Nachr. 18 (1958), 236-243.

[He] A. Heppes, On the densest packing of discs of radius 1 and $\sqrt{2}-1$, Studia Sci. Math. Hungar. 36 (2000), 433-454.

[HM] A. Heppes and J. Molnár, Újabb eredmények a diszkrét geometriában I, Mat. Lapok 11 (1960), 330_ 355 (in Hungarian).

[Mo] J. Molnár, Unterdeckung und Überdeckung der Ebene durch Kreise, Ann. Univ. Sci. Budapest, 2 (1959), 33-40.

[Th1] A. Thue, Om Nogle Geometrisk Taltheoretiske Theoremer, Forhdl. Skand. Naturforsk. 14 (1892), 352-353.

[Th2] A. Thue, Über die dichteste Zusammenstellung von kongruenten Kreisen in der Ebene, Christiania Vid. Selsk. Skr. 1 (1910), 3-9.

Received September 18, 2000, and in revised form September 17, 2001, April 30, 2002, and September 18, 2002. Online publication July 15, 2003. 